\title{
Análisis comparativo de los atractivos históricos arqueológicos antes y después del terremoto en del cantón San Vicente
}

\section{Comparative analysis of the historical archaeological attractions before and after the earthquake in San Vicente canton}

\section{Análisis comparativo de los atractivos históricos arqueológicos}

\author{
Carlos Chica Medranda ${ }^{(1)}$ \\ Oscar Cedeño Falconí( ${ }^{(2)}$ \\ Lilia Villacis Zambrano ${ }^{(3)}$ \\ William Meneses Pantoja ${ }^{(4)}$
}

(1) Universidad Laica Eloy Alfaro de Manabí, Ecuador. email: bahiadolphin@gmail.com

(2) Universidad Laica Eloy Alfaro de Manabí, Ecuador.

(3) Universidad Laica Eloy Alfaro de Manabí, Ecuador.

(4) Universidad Laica Eloy Alfaro de Manabí, Ecuador.

Contacto: bahiadolphin@gmail.com

Recibido: 19-05-2020

Aprobado: 08-06-2020

\section{Resumen}

La presente investigación tiene por objeto el análisis comparativo de los atractivos históricosarqueológicos del cantón San Vicente, Ecuador. La tendencia dentro de los últimos años es la puesta en valor del patrimonio arqueológico, lo que ha permitido la accesibilidad a una buena parte de los recursos, con una proyección social, educativa y cultural, no solo dentro del mercado interno sino incluso del mercado internacional. Esta disciplina presenta una dualidad práctica: estudia el pasado a través de los restos materiales y a la vez los preserva para futuras generaciones. En las últimas décadas, la Arqueología ha ampliado extraordinariamente sus fronteras en muchas direcciones, pero dos parecen las más importantes: la Gestión del Patrimonio Arqueológico en todas sus dimensiones y la enseñanza e investigación en las universidades. La sociedad ha desarrollado un interés especial por el pasado y el patrimonio histórico de Manabí, El presente trabajo es el resultado del proyecto de investigación de la ruta histórica - arqueológica de los cantones Sucre, San Vicente, Jama y Pedernales, de la Universidad Laica Eloy Alfaro de Manabí, donde el objetivo fundamental es la creación de nuevos espacios para el turismo, la educación y la investigación. La metodología que se aplico fue análisis de Kendall, donde se identifica 10 lugares arqueológicos, los mismos no han sido jerarquizados por el Mintur o el GAD de San Vicente, estos espacios podrían convertirse en recursos turísticos potenciales, requiriendo la presencia de profesionales que sepan atender las diversas vertientes que entraña este tipo de oferta cultural.

Palabras claves: atractivos, históricas, arqueológicas, terremoto, comparativo.

\begin{abstract}
The purpose of this research is the comparative analysis of the historical - archaeological attractions of the San Vicente canton, Ecuador. The trend in recent years is the enhancement of archaeological heritage, which has allowed access to a good part of the resources, with a social, educational and cultural projection, not only within the internal market but also the international market. This discipline presents a practical duality: it studies the past through material remains and at the same time preserves them for future generations. In recent decades, Archeology has greatly expanded its borders in many directions, but two seem the most important: the Management of Archaeological Heritage in all its dimensions and teaching and research in universities. The society has developed a special interest for the past and the
\end{abstract}


historical heritage of Manabí, This work is the result of the research project of the historical archaeological route of the Sucre, San Vicente, Jama and Pedernales cantons, of the Eloy Lay University Alfaro de Manabí, where the fundamental objective is the creation of new spaces for tourism, education and research. The methodology that was applied was Kendall's analysis, where 10 archaeological sites are identified, they have not been hierarchized by the Mintur or the GAD of San Vicente, these spaces could become potential tourist resources, requiring the presence of professionals who know how to attend the different aspects that this type of cultural offer entails.

Keywords: attractive, historical, archaeological, earthquake, comparative

\section{Introducción.}

El presente proyecto es conseguir un análisis comparativo de los atractivos históricos arqueológicos del cantón San Vicente. En los últimos años los proyectos de investigación y puesta en valor del patrimonio arqueológico han permitido la accesibilidad a una buena parte de los recursos arqueológicos, con una proyección social, educativa y cultural, no solo dentro del mercado interno sino incluso del mercado internacional (Tresserras, 2004).

Partiendo el concepto de arqueoturismo o turismo arqueológico, se considera una modalidad de turismo cultural bajo la que se presentan propuestas turísticas con la arqueología como ingrediente principal. Esta fórmula turística alternativa a lo convencional está basada en promocionar la pasión por la arqueología y las iniciativas de conservación de los yacimientos y lugares históricos. El patrimonio no ha sido ajeno al turismo y ha sido uno de los primeros motivos de compra de este tipo de viajes. Aún incluso de que se pudiera hablar de turismo tal como se entiende actualmente. Asimismo, el patrimonio cultural, desde hace mucho tiempo, constituye una razón capaz de generar desplazamientos de personas con intereses específicos (Romero, 2015).

Hoy, cuando se entrelazan en una trama muy compleja los procesos de globalización, con la reivindicación de las singularidades regionales y la búsqueda de los orígenes, la Arqueología se ha hecho más popular que nunca. Esta disciplina presenta una dualidad práctica: estudia el pasado a través de los restos materiales y a la vez los preserva para futuras generaciones. En las últimas décadas, la Arqueología ha ampliado extraordinariamente sus fronteras en muchas direcciones, pero dos parecen las más importantes en este sentido: la Gestión del Patrimonio Arqueológico en todas sus dimensiones y la enseñanza e investigación en las universidades y centros de investigación.

La sociedad ha desarrollado un interés especial por el pasado y el patrimonio que éste nos ha legado, tanto en sus aspectos más generales como por los testimonios vinculados a cada contexto territorial, en tanto que elemento que ayuda a definir o vertebrar la identidad cultural. Esta circunstancia se ha traducido en la multiplicación de publicaciones científicas, divulgativas e incluso de ficción que apelan a esa reconstrucción del pasado, cobrando gran protagonismo las disciplinas que se emplean en esa tarea, especialmente la Arqueología. Junto al interés editorial, se observa un aumento de la presencia de espacios dedicados a preservar vestigios del pasado (Museos de sitio, parques arqueológicos), que se han venido a unir a los tradicionales museos, cumpliendo funciones de conservación, enseñanza y difusión de todo ese patrimonio cultural. Estos espacios se han convertido asimismo en recursos turísticos y aulas docentes, requiriendo la presencia de profesionales que sepan atender las diversas vertientes que entraña este tipo de oferta cultural (Domínguez, Chacón, Mejía, Sánchez, y Ibarra,2003).

Basándose en esta realidad se propuso como objetivo el análisis comparativo de los atractivos históricosarqueológicos del cantón San Vicente.

\section{Materiales y métodos}

El trabajo investigativo estuvo centrado en el método cuantitativo -cualitativo, se realizó una encuesta a 10 sujetos con el método de Kendall para expertos en el tema de historia y arqueología en el Cantón San Vicente, Ecuador.

La finalidad de los resultados es analizar el complejo mundo de la arqueología y la reconstrucción histórica a partir de restos materiales de sociedades ancestrales que ocuparon la zona en investigación, en este caso San Vicente. Por lo tanto, esta técnica de investigación proporciona información viable de datos y experiencia provenientes de la realidad actual y a partir de los resultados obtenidos se comprobará con certeza si en realidad estos lugares que fueron identificados 10 en la zona, son recursos que pueden convertirse en el corto tiempo en productos turísticos relevantes para mejorar la afluencia de visitantes hacia este cantón. 


\section{Concepto de turismo cultural}

El turismo cultural apela a la creación y a la memoria del hombre, al testimonio de su paso por la tierra, a su historia. Si entendemos por cultura todo aquello que ha sido transformado por el hombre, se podría considerar turismo cultural no sólo aquél que es atraído por la obra de arte, el museo o los monumentos, sino también al turismo de naturaleza, al paisaje transformado durante siglos por el hombre. Habría así un patrimonio religioso, un patrimonio civil (castillos, palacios, museos), un patrimonio arqueológico, un patrimonio industrial y un humilde pero no menos interesante y en vías de desaparición, totalmente despreciado patrimonio agrícola (viejas granjas, tinadas, construcciones rurales y pastoriles, etc. (Oyarzún, 1998).

Sierra (2014), afirma que este tipo de turismo precisa de recursos histórico-artísticos para su desarrollo, Ecuador cuenta con ciudades llenas de historia y cultura propia, concentrada en museos, construcciones, costumbres, tradiciones, atracciones, comidas etc. El turismo cultural es una especie de turismo más exigente y menos estacional, con una duración de 3 a 4 días. Ecuador es cultura, historia, paraísos naturales y mucho más.

El turismo cultural aparece en las cuatro últimas décadas 9 como un fenómeno social que presenta una de las mejores perspectivas para ocupar el tiempo libre, vinculado con la herencia histórica de los territorios en donde se desarrolla, contribuyendo al desarrollo regional y de las ciudades y poblados que son depositarios de un importante legado patrimonial. Al respecto Tomás Villasante Rodríguez manifiesta: Turismo y cultura son dos realidades destinadas a converger en sus objetivos, intereses y programas y a coordinar su potencial y su capacidad de atracción de visitantes, a partir de lo que conocemos como turismo cultural, un concepto que responde, en la actualidad, a una nueva demanda social y supone un alto consumo de servicios, que debe revertir en beneficio tanto del patrimonio y su conservación, como en el desarrollo socioeconómico de la zona de atracción turística (Pardo, 2011).

No cabe duda que el turismo cultural "es todo aquello transformado por el hombre" señala Valdivia, 1998; El concepto de turismo cultural ha ido variando con los años, Las múltiples opciones y perspectivas de lo que se entiende por cultura impide el acto de definir. Y lo que se interpreta por turismo cultural va ligado la antigüedad y la arqueología que en muchos casos está concentrada en museos y centros históricos a nivel global, estas construcciones permiten que el hombre pueda almacenar los cambios constantes de los seres humanos en todas sus dimensiones como costumbres, tradiciones, atracciones, comidas folclor, lengua, entre otros etc.

\section{La conferencia mundial sobre la cultura y el turismo}

La Cuarta Conferencia Mundial sobre la Cultura y el Turismo de la Organización Mundial del Turismo (OMT, 2018) y la Organización de las Naciones Unidas para la Educación, la Ciencia y la Cultura (UNESCO, 2019) del 13 de diciembre del 2019 pone de relieve el valor añadido de la cultura en los destinos y se centra en la futura sostenibilidad del turismo cultural. Varios delegados han debatido en la capital de la cultura del Japón la manera de mantener en el centro del turismo, en beneficio de las generaciones venideras, la valoración del patrimonio y la expresión cultural contemporánea. El diálogo intercultural, las comunidades locales y los sistemas innovadores de medición ocupan un lugar destacado en las conclusiones de la Conferencia, expuestas en la Declaración de Kyoto (UNTWO, 2020).

Manuel Butler, Director Ejecutivo de la OMT, afirmó que: "Un turismo cultural gestionado de forma responsable puede enriquecer la vida de los visitantes y los residentes al promover la diversidad y el diálogo intercultural. La Declaración de Kyoto nos ayudará a velar por que el rico patrimonio y la diversidad creativa de nuestro mundo sean maravillas que nuestros hijos también tengan oportunidad de descubrir por cuenta propia". Xing Qu, Subdirector General de la UNESCO, agregó que: "La comunidad internacional debe percibir los beneficios de conectar la cultura y el turismo como fuerzas mundiales que aúnan a los pueblos. Las prácticas responsables ocupaban el primer plano en las soluciones propuestas por destacados expertos en la materia, como la inclusión de la población local en todos los niveles del desarrollo del turismo cultural, el uso de nuevas tecnologías para gestionar de forma sostenible las corrientes de visitantes y la distribución equitativa de los beneficios del turismo.

Durante los últimos años se han realizado una serie de actividades con el propósito de unificar estrategias a favor del desarrollo turístico en un marco de respeto por la cultura y la naturaleza. Uno de los productos de esta estrecha colaboración es el Programa de Patrimonio Mundial y Turismo Sostenible cuya misión es facilitar la gestión y el desarrollo del turismo sostenible en los bienes del Patrimonio Mundial a través de fomentar una mayor conciencia, la capacidad y la participación equilibrada de todas 
las partes interesadas a fin de proteger las propiedades y su valor universal excepcional. (UNESCO, 2020).

En relación al ecoturismo actualmente los países con menor desarrollo industrial, aún poseen una riqueza natural que les permite ofertar este tipo de actividad, especialmente aquellos que se han preocupado por establecer y proteger áreas con población endémica, es así que alrededor del mundo las zonas de mayor diversidad (hotspots), atraen el interés del mundo ya que sólo queda un 5\% de la superficie total del planeta. Es por esta razón que el ecoturismo según la OMT, (2012) presenta uno de los crecimientos más altos con un porcentaje del $15 \%$ anual (UNTWO, 2020).

\section{Concepto de arqueología}

La palabra Arqueología deriva de dos términos: Arqueos, que designa el pasado, lo antiguo y Logos que se trata del estudio, discurso tratado etc. Podemos definir la Arqueología como "la ciencia social que estudia las culturas del pasado mediante el análisis e interpretación de los diversos tipos de vestigios materiales que toda actividad humana genera". Según Fraind (2015), asevera que una definición clásica para la arqueología, es que se concibe como la recuperación, descripción y estudio sistemáticos de la cultura material del pasado como forma de acceder a las sociedades que la construyeron. Nosotros la definimos como el estudio de la Historia a partir de los aspectos no hablados ni escritos de la cultura material. Generalmente, para definir la arqueología se suelen utilizar expresiones negativas (qué no es la arqueología) o expresiones metodológicas (qué significa la palabra arqueología); en un sentido etimológico, la arqueología es la ciencia que se ocupa del estudio de lo antiguo (Maripaz, 2013).

España se encuentra entre los primeros diez destinos arqueoturísticos según un estudio realizado por la Universitat de Barcelona y la Red Ibertur para la Asociación Española de Expertos Científicos en Turismo (AECIT) en el 2009. Ocupa el sexto lugar. Italia, Egipto, Grecia, México y Perú están por delante. A su vez, Catalunya, Andalucía, Cantabria y Madrid son los principales destinos del país. Y los considerados más atractivos y con más visitas anuales los siguientes: los museos integrados en el arqueoticket de Barcelona, los conjuntos arqueológicos de Tarragona (502.430 visitas al año) y Mérida (415.766) con la Vía de la Plata, el teatro de Málaga, el Museo de la Alhambra, el Museo y la Neocueva de Altamira, el Museo Arqueológico
Nacional, el yacimiento grecorromano de Empúries (l'Escala, 328.161 visitas), integrado en el Museu d'Arqueologia de Catalunya, y el conjunto de Madinat al-Zahra en Córdoba. Se suma el acueducto de Segovia (Talavera, 2020).

\section{El arqueoturismo o turismo arqueológico}

El Arqueoturismo o Turismo Arqueológico es una modalidad bajo la que se presentan propuestas y productos culturales y turísticos en los que la arqueología es el ingrediente principal. Cierto es que en el imaginario cuando se habla de turismo arqueológico vienen a la mente imágenes de Egipto, Grecia, Italia, Túnez, Turquía, México o Perú (Tresserras,2004). A nivel centro y sur América, Perú y México se han posicionado fuertemente en el turismo de arqueología con sus productos estrellas como Machu Pichu, y las pirámides Mayas, pero en el mercado internacional a Ecuador no se lo asocia como un destino de turismo arqueológico, por lo tanto es un producto que requiere de mayor atención por parte de las autoridades del Ministerio de Turismo y Patrimonio Cultural del Ecuador para afianzar un recurso turístico en un producto de primer orden a nivel internacional (Estrada, 1962).

Los proyectos de investigación en arqueología para la costa del Ecuador han sido significativos, abriendo puertas a sitios que antes eran totalmente desconocido por los ecuatorianos y los propios extranjeros, la ley de patrimonio cultural del estado, permite y garantiza la puesta en valor del patrimonio arqueológico con fines educativos, turísticos y de investigación, que los lugares donde se encuentran las muestras arqueológicas tengan los requerimientos mínimos y estándares de accesibilidad a una buena parte de los recursos arqueológicos, el turismo de los últimos 5 años ha tenido un giro radical y con una proyección, no sólo dentro del mercado interno sino incluso del mercado internacional.

\section{Resultados}

En las tablas 1 (a y b), se aprecia la importancia de las jerarquías está dada mediante descripciones que delimitan el nivel de impacto turístico sobre una zona, región o área determinada. Jerarquía I y II presentan condiciones mínimas o llamativas para generar productos turísticos, jerarquía III y IV son atractivos con rasgos excepcionales y de alto consumo turístico, pues se venden por si solos y motivan al desplazamiento del viajero. 


\begin{tabular}{|l|l|l|l|}
\hline Cantón San Vicente & Parroquia & Sitios Naturales & $\begin{array}{l}\text { Manifestaciones } \\
\text { culturales. }\end{array}$ \\
\cline { 2 - 4 } & San Vicente & 3 & 3 \\
\cline { 2 - 4 } & San Andrés de Canoa & 5 & 1 \\
\cline { 2 - 3 } & Total & 8 & 4 \\
\hline
\end{tabular}

Tabla 1a. Lugares turísticos

\begin{tabular}{|c|c|c|c|c|c|}
\hline Parroquia & Categoría & Recurso & Jerarquía & $\begin{array}{l}\text { Recurso } \\
\text { actual/potencial }\end{array}$ & Habilitado \\
\hline San Vicente & $\begin{array}{l}\text { Sitios Naturales } \\
\text { (P/A) }\end{array}$ & Playa Pedernales & 2 & Actual & Sí \\
\hline San Vicente & $\begin{array}{l}\text { Sitios Naturales } \\
\text { (P/A) }\end{array}$ & Playa Punta Napo & 1 & Actual & Sí \\
\hline San Vicente & $\begin{array}{l}\text { Sitios Naturales } \\
(\mathrm{P} / \mathrm{A})\end{array}$ & $\begin{array}{l}\text { Refugio de vida silvestre } \\
\text { "Isla Corazón y Fragatas" }\end{array}$ & 3 & Actual & Sí \\
\hline San Vicente & $\begin{array}{l}\text { Manifestaciones } \\
\text { culturales (Año) }\end{array}$ & Fiestas patronales & 2 & Potencial & P/A \\
\hline San Vicente & $\begin{array}{l}\text { Manifestaciones } \\
\text { culturales (Año) }\end{array}$ & $\begin{array}{l}\text { Iglesia Santa Rosa de } \\
\text { Lima }\end{array}$ & 3 & Actual & Sí \\
\hline San Vicente & $\begin{array}{l}\text { Manifestaciones } \\
\text { culturales (Año) }\end{array}$ & $\begin{array}{l}\text { Fiesta San Pedro y San } \\
\text { Pablo }\end{array}$ & 3 & Potencial & $\mathrm{P} / \mathrm{A}$ \\
\hline Canoa & $\begin{array}{l}\text { Sitios Naturales } \\
(\mathrm{P} / \mathrm{A})\end{array}$ & Playa Canoa & 2 & Actual & Sí \\
\hline Canoa & $\begin{array}{l}\text { Sitios Naturales } \\
\text { (P/A) }\end{array}$ & Cabo Pasado & 2 & Potencial & Sí \\
\hline Canoa & $\begin{array}{l}\text { Sitios Naturales } \\
\text { (P/A) }\end{array}$ & Los Aposentos & 2 & Actual & Sí \\
\hline Canoa & $\begin{array}{l}\text { Sitios Naturales } \\
(\mathrm{P} / \mathrm{A})\end{array}$ & Puerto Cubuyal & 2 & Potencial & Sí \\
\hline Canoa & $\begin{array}{l}\text { Sitios Naturales } \\
(\mathrm{P} / \mathrm{A})\end{array}$ & $\begin{array}{l}\text { Parque temático Río } \\
\text { Muchacho }\end{array}$ & 2 & Actual & Sí \\
\hline Canoa & $\begin{array}{l}\text { Manifestaciones } \\
\text { culturales (Año) }\end{array}$ & $\begin{array}{l}\text { Cerveza artesabal } \\
\text { BeerKingo }\end{array}$ & 2 & Actual & Sí \\
\hline
\end{tabular}

Tabla 1b. Lugares turísticos jerarquizados.

Fuente: Estudiantes de la ULEAM. Octavo año de Licenciatura en Turísmo. Extensión Bahia de Caraquez. Navas, (2018).

Los resultados reflejan que en la tabla comparativa primero se procede a comparar la fuente de resultados que fue tomada del observatorio turístico de la Uleam y se analiza los resultados del trabajo de campo de jerarquización durante el 2018. En el caso del cantón San Vicente cuenta con tres (3) atractivos de jerarquía III, ocho (8) de jerarquía II y uno (1) de jerarquía I, a pesar de tener un menor número de atractivos turísticos dentro del territorio, es un destino posesionado en el mercado nacional y extranjero ya que motivan al desplazamiento del viajero por sí solo, se observa en la tabla 2. 


\begin{tabular}{|c|c|c|c|c|c|c|}
\hline \# & Parroquia & Categoría & $\begin{array}{l}\text { Nombre del } \\
\text { recurso }\end{array}$ & Jerarquía & $\begin{array}{l}\text { Recurso } \\
\text { actual/potencial }\end{array}$ & Habilitado \\
\hline 1 & San Vicente & $\begin{array}{l}\text { Manifestaciones } \\
\text { culturales }\end{array}$ & $\begin{array}{l}\text { Restos de un } \\
\text { Mastodonte }\end{array}$ & 1 & Potencial & No \\
\hline 2 & Cabo pasado & $\begin{array}{l}\text { Manifestaciones } \\
\text { culturales }\end{array}$ & $\begin{array}{l}\text { Corrales de pesca } \\
\text { precolombinos }\end{array}$ & 1 & Potencial & No \\
\hline 3 & \begin{tabular}{|l|} 
Rio \\
Muchacho \\
\end{tabular} & $\begin{array}{l}\text { Manifestaciones } \\
\text { culturales }\end{array}$ & \begin{tabular}{|l|} 
Tolas \\
arqueológicas
\end{tabular} & 1 & Potencial & $\mathrm{Si}$ \\
\hline 4 & San Vicente & $\begin{array}{l}\text { Manifestaciones } \\
\text { culturales }\end{array}$ & $\begin{array}{l}\text { Rosa Blanca } \\
\text { restos } \\
\text { arqueológicos }\end{array}$ & 1 & Potencial & No \\
\hline 5 & Rio Briceño & $\begin{array}{l}\text { Manifestaciones } \\
\text { culturales }\end{array}$ & $\begin{array}{l}\text { San Miguel de } \\
\text { Briceño resto } \\
\text { arqueológicos }\end{array}$ & 1 & Potencial & No \\
\hline 6 & Canoa & $\begin{array}{l}\text { Manifestaciones } \\
\text { culturales }\end{array}$ & $\begin{array}{l}\text { Cuevas } \\
\text { comunicantes y } \\
\text { petroglifos } \\
\end{array}$ & 1 & Potencial & No \\
\hline 7 & Muyuyal & $\begin{array}{l}\text { Manifestaciones } \\
\text { culturales }\end{array}$ & $\begin{array}{l}\text { San Miguel de } \\
\text { Briceño resto } \\
\text { arqueológicos }\end{array}$ & 1 & Potencial & No \\
\hline 8 & Cabuyal & $\begin{array}{l}\text { Manifestaciones } \\
\text { culturales }\end{array}$ & \begin{tabular}{|l} 
San Miguel de \\
Briceño resto \\
arqueológicos
\end{tabular} & 1 & Potencial & No \\
\hline 9 & La Mocora & $\begin{array}{l}\text { Manifestaciones } \\
\text { culturales }\end{array}$ & $\begin{array}{l}\text { San Miguel de } \\
\text { Briceño resto } \\
\text { arqueológicos }\end{array}$ & 1 & Potencial & No \\
\hline 10 & Salinas & $\begin{array}{l}\text { Manifestaciones } \\
\text { culturales }\end{array}$ & $\begin{array}{l}\text { San Miguel de } \\
\text { Briceño resto } \\
\text { arqueológicos }\end{array}$ & 1 & Potencial & No \\
\hline
\end{tabular}

Tabla 2. GAD San Vicente y proyecto histórico - arqueológico Uleam 2020

Al comparar los resultados con el trabajo realizado en 2018, se puede constatar que no se ha realizado el trabajo de la aplicación de la ficha metodológica del Mintur sobre los sitios o lugares que tienen restos de evidencias arqueológicas dentro del cantón San Vicente, después de este periodo de jerarquización 2018, en el proyecto histórico -Arqueológico se ha encontrado algunos lugares con importante restos de cerámica que pertenece a la época indígena del Ecuador precolombino, lo que hace suponer de la importante que tienen estos estos sitios para la arqueología, la investigación y el turismo, que conllevara a seguir investigando desde otros indicadores que el mismo proyecto propone, que será a posteriori.

\section{Discusión}

Revisando los resultados del trabajo del método de Kendall para expertos en el tema de historia y arqueología, se puede observar que los resultados 0,06 demuestra que existe muy poco conocimiento o interés sobre los lugares que tienen restos 0 evidencias de arqueología en el Cantón San Vicente, pues se sugiere que una vez identificado los 10 lugares que tienen restos de arqueología independientemente de la cultura a la que pertenezcan, son un verdadero potencial para intereses turísticos de la zona en mención, por lo que es fundamental realizar un trabajo de jerarquización utilizando la metodología del ministerio de turismo, para determinar de los 10 sitios identificados, cual es ellos o cuántos de ellos pueden entrar en un plan de elaboración de producto turístico novedoso que impacte al mercado nacional e internacional con fines turísticos. 
En este estudio se concuerda con Campos, Martí y Mejía (2017), señalan la efectividad de diversas estrategias orientadas a la reactivación de los destinos turísticos, utilizando factores claves como la solidaridad y los recursos turísticos propios de la identidad manabita no condicionada por infraestructuras afectadas, permitieron volver a posicionar Manabí como la segunda provincia más visitada a nivel nacional. Si bien se reflejan cambios de tendencia a corto plazo como respuesta positiva a las estrategias inmediatas de contención tras la catástrofe esto puede contribuir a cambios en el tipo de producto que se ofrece en la provincia y su gestión de destino como tal.

También se coincide con otras investigaciones realizadas por Carvajal y Lemoine (2018), aseveran que San Vicente posee gran potencial para un mejor desarrollo como sitio de interés turístico, tanto para visitantes nacionales como extranjeros.

Hasta ahora el que tiene mayor mención y que ha sido utilizado por operadoras de turismos es la visita de los corrales marinos ancestrales, de la cual se ha escrito algunos trabajos en investigaciones recientes en la zona costera de Manabí, estos corrales se encuentran en el cabo Pasado, por lo que su acceso es solo en bote o vía terrestre pero no época de lluvia, por lo que se requiere de una intervención para su protección y quien sabe algún tipo de declaratoria de patrimonio cultural para este cantón, se concuerda con los estudios de (Navas, 2018).

\section{Conclusiones}

Se concluye que por ahora lo único que se conoce en el cantón San Vicente es el hallazgo de resto del mastodonte en el sitio la Unión, y que por información interna del GAD, se ha escuchado de la posibilidad de reconstruir un museo donde se pueda exhibir esta importante pieza de arqueología.

Los resultados también demuestran que no se ha jerarquizado por el momento ninguno de los 10 lugares identificado con una fuerte presencia arqueológica en la zona de San Vicente, lo que requiere de un nuevo estudio para su próxima proyección con fines turísticos

Según el análisis en el método de Kendall, se puede apreciar que los corrales submarinos son un verdadero tesoro que está guardado en el Cabo pasado, y que en el corto tiempo representa un potencial para el turismo histórico - arqueológico de San Vicente.

\section{Bibliografía}

Campos, Martí y Mejía. (2017). Recuperación de destinos turísticos posterremoto: revisión del caso. Ecuador. Revista Venezolana de Gerencia 22, 80. Recuperado de https://www.redalyc.org/jatsRepo/290/290 55967006/html/index.html.

Carvajal, G. y Lemoine, F. (2018). Análisis de los atractivos y recursos turísticos del cantón San Vicente. Revista el Periplo Sustenatble, 34 .

Domínguez, V; Chacón, R; Mejía, F; Sánchez, F; y Ibarra, A. (2003). Identificación de zonas arqueológicas en el área urbana de Quito. Quito: Fondo de Salvamento.

Estrada, E. (1962). Arqueología de Manabì Central, Guayaquil: Publicaciones Victor Emilio Estrada,

205.

Félix, A., Campos, S., Martí, J. y Mejía, M. (2017). Recuperación de destinos turísticos posterremoto: revisión del caso Ecuador. Revista Venezolana de Gerencia, 22, (80). Recuperado de: https://www.redalyc.org/jatsRepo/290/290 55967006/html/index.html

Fraind, E. (2015).Arqueología conceptos básicos. Recuperado de: https://www.studocu.com/esdocument/univ ersidad-complutense-madrid/teoriaarqueologica/apuntes/arqueologiaconceptos-basicos/1937590/view

Maripaz. (2013). Definición y evolución del concepto de arqueología. Recuperado de: http://miarqueologia.blogspot.com/2013/06 /definicion-y-evolucion-del-concepto$\underline{\text { de.html }}$

Navas, F. (2018). Uso de la metodologia Mintur para la actualizacion de los atractivos turisticos de la zona norte. En Uleam. Manta: Mar abierto.

Oyarzún M., E. (1998). El turismo cultural, potencialidades y riesgos en la region de los lagos, Chile. Gestión Turística, (2), 75-89. doi:10.4206/gest.tur.1998.n2-03 
Pardo, C. (2011). Panorama mundial del turismo cultural. Cuadernos de Patrimonio Mundial y Turismo. Recuperado de: https://www.cultura.gob.mx/turismocultura 1/cuadernos/pdf18/articulo1.pdf

Romero, A. (2015). Arqueoturismo, patrimonio arqueológico como recurso turístico. Rural Empresarial. Recuperado de: http://www.ruralempresarial.com/arqueotur ismo/

Sierra, J. (2014). Turismo Cultural. Portal Web vivenuestromundo.com. Recuperado de: https://www.vivenuestromundo.com/2014/ $\underline{03 / 24 / \text { turismo-cultural-2/ }}$

Talavera, A. (2020). Turismo cultural, culturas turísticas. Revista Horiz. Antropol. 9 (20). Recuperado http://www.scielo.br/scielo.php?script=sci $\underline{\text { arttext\&pid=S0104-7183320000200003 }}$

https://www.unwto.org/es/unwto-tourism-dashboard.
Tresserras, J. (2004). El arqueoturismo o turismo arqueológico: un paso más para la valorización del patrimonio arqueológico. Portal Iberoamericano de Gestión Cultural para su publicación en el Boletín GC: Gestión Cultural, 9. Recuperado de: https://web.archive.org/web/200610091621 24/http://www.gestioncultural.org/gc/boleti n/pdf/Arqueoturismo/JJuan.pdf

UNESCO. (2020). Conservación de las identidades culturales en aras del futuro del turismo.

Recuperado de www.unesco.org

UNTWO, O. (2020). World Tourism Organization. Recuperado de http://www2.unwto.org/es

UNTWO. (2020). The UNWTO Tourism Dashboard - insights on key performance indicators for inbound and outbound tourism at the global, regional and national levels. Sustainable Tourism for Development Guidebook. Recuperado. 